\title{
Non-normal very ample polytopes and their holes
}

\author{
Akihiro Higashitani* \\ Department of Pure and Applied Mathematics \\ Graduate School of Information Science and Technology \\ Osaka University, Japan \\ a-higashitani@cr.math.sci.osaka-u.ac.jp
}

Submitted: Aug 16, 2013; Accepted: Feb 26, 2014; Published: Mar 10, 2014

Mathematics Subject Classifications: 52B20; 14M25; 52B12

\begin{abstract}
In this paper, we show that for given integers $h$ and $d$ with $h \geqslant 1$ and $d \geqslant 3$, there exists a non-normal very ample integral convex polytope of dimension $d$ which has exactly $h$ holes.
\end{abstract}

\section{Introduction}

The normality and the very ampleness of integral convex polytopes are of importance in several points of view, e.g., not only combinatorics on convex polytopes but also toric geometry and commutative algebra. In particular, normal or very ample integral convex polytopes appearing in the context of toric geometry are well studied (cf. [1, 2, 3, 8, 9, 10]). To determine whether a given integral convex polytope is normal (very ample) or not is a fascinating problem. (See $[7,11,12]$.) In this paper, we will show the existence of non-normal very ample integral convex polytopes with an additional property in any dimension.

Let $\mathcal{P} \subset \mathbb{R}^{d}$ be an integral convex polytope, which is a convex polytope all of whose vertices are contained in $\mathbb{Z}^{d}$, of dimension $d$. Define $\widetilde{\mathcal{P}} \subset \mathbb{R}^{d+1}$ to be the convex hull of the points $(\alpha, 1) \in \mathbb{R}^{d+1}$ with $\alpha \in \mathcal{P}$ and let $\mathcal{A}_{\mathcal{P}}=\widetilde{\mathcal{P}} \cap \mathbb{Z}^{d+1}$. We say that $\mathcal{P}$ is normal if $\mathcal{P}$ satisfies

$$
\mathbb{R}_{\geqslant 0} \mathcal{A}_{\mathcal{P}} \cap \mathbb{Z}_{\mathcal{P}}=\mathbb{Z}_{\geqslant 0} \mathcal{A}_{\mathcal{P}}
$$

Moreover, we say that $\mathcal{P}$ is very ample if the set

$$
\left(\mathbb{R}_{\geqslant 0} \mathcal{A}_{\mathcal{P}} \cap \mathbb{Z} \mathcal{A}_{\mathcal{P}}\right) \backslash \mathbb{Z}_{\geqslant 0} \mathcal{A}_{\mathcal{P}}
$$

\footnotetext{
*The author is supported by JSPS Research Fellowship for Young Scientists.
} 
is finite and we call the elements of $\left(\mathbb{R}_{\geqslant 0} \mathcal{A}_{\mathcal{P}} \cap \mathbb{Z} \mathcal{A}_{\mathcal{P}}\right) \backslash \mathbb{Z}_{\geqslant 0} \mathcal{A}_{\mathcal{P}}$ the holes of $\mathcal{P}$. (In [1], holes are called gaps.) In particular, when $\mathcal{P}$ is normal, $\mathcal{P}$ is very ample.

In addition, for a positive integer $k$, we say that $\mathcal{P}$ is $k$-normal if for each $n=k, k+1, \ldots$ and for each $\alpha \in n \mathcal{P} \cap \mathbb{Z}^{d}$, where $n \mathcal{P}=\{n \alpha: \alpha \in \mathcal{P}\}$, there exist $n$ integer points $\alpha_{1}, \ldots, \alpha_{n}$ belonging to $\mathcal{P} \cap \mathbb{Z}^{d}$ such that $\alpha=\alpha_{1}+\cdots+\alpha_{n}$.

Let us assume

$$
\mathbb{Z} \mathcal{A}_{\mathcal{P}}=\mathbb{Z}^{d+1}
$$

Then $\mathcal{P}$ is normal if and only if $\mathcal{P}$ is 1 -normal. Moreover, $\mathcal{P}$ is very ample if and only if $\mathcal{P}$ is $k$-normal for some sufficiently large positive integer $k$. A definition of very ampleness described in $[5,6,9]$ is equivalent to ours ([1, Proposition 2.1]).

The notions "normal" or "very ample" also arise naturally in the contex of toric varieties. For an algebraically closed filed $\mathbb{K}$ and an integral convex polytope $\mathcal{P}$, we write $\mathbb{K}\left[\mathbb{Z}_{\geqslant 0} \mathcal{A}_{\mathcal{P}}\right]$ for the toric ring arising from $\mathcal{P}$. Let $X_{\mathcal{P}}=\operatorname{Proj}\left(\mathbb{K}\left[\mathbb{Z}_{\geqslant 0} \mathcal{A}_{\mathcal{P}}\right]\right) \subset \mathbb{P}_{\mathbb{K}}^{N-1}$, where $N$ is the number of integer points in $\mathcal{P}$. Then $\mathcal{P}$ is normal if and only if the corresponding projective variety $X_{\mathcal{P}}$ is projectively normal, i.e., its affine cone is normal. Moreovr, $\mathcal{P}$ is very ample if and only if $X_{\mathcal{P}}$ is normal. For more detailed information, consult, e.g., $[5$, Chapter 2] and [6, Chapter 2].

It often happens that for some class of integral convex polytopes, its normality is equivalent to its very ampleness. For example, edge polytope is a typical example (cf [11]). Thus, the following is a quite natural question:

Does there exist an integral convex polytope which is not normal but very ample?

In [4, Example 5.1], Bruns and Gubeladze succeeded in giving the first example of a non-normal very ample integral convex polytope, which is of dimension 5 and can be obtained from a triangulation of a real projective plane. Recently, they provided the second example in [5, Exercise 2.24], which is of dimension 3. Moreover, in [9, Section 2], Ogata generalized the second example and established infinitely many non-normal very ample integral convex polytopes of dimension 3 .

In this paer, we present a non-normal very ample integral convex polytope in any dimension having an additional property. The following is our main theorem of this paper.

Theorem 1. Let $h$ and $d$ be integers with $h \geqslant 1$ and $d \geqslant 3$. Then there exists a 3-normal but non-normal integral convex polytope of dimension $d$ which has exactly $h$ holes.

Remark 2. In the 3-dimensional case, the existence of non-normal very ample integral convex polytopes having exactly $h$ holes is known in [2, Example 15]. This example is exhaustively analyzed in [1, Theorem 3.2].

Remark 3. Let $\mathcal{P}$ be an integral convex polytope which is not normal but very ample. Then $\mathcal{P} \times[0,1]$ is also very ample by [6, Theorem 2.4.7]. Moreover, $\mathcal{P} \times[0,1]$ is not normal. Thus, $\mathcal{P} \times[0,1]$ is non-normal and very ample, in particular, $\mathcal{P} \times[0,1]^{n}$ is a nonnormal very ample integral convex polytope of dimension $\operatorname{dim} \mathcal{P}+n$. Hence, we know 
the existence of non-normal very ample integral convex polytopes in any dimension at least 3. However, we cannot show Theorem 1 by using this inductive construction. More precisely, the number of holes of $\mathcal{P} \times[0,1]$ is not equal to that of $\mathcal{P}$. In fact, one sees that the number of holes of $\mathcal{P} \times[0,1]$ is equal to $\sum_{k \geqslant 2}(k+1) h_{k}$, where $h_{k}$ is the number of holes of $\mathcal{P}$ with degree $k$.

Let $h$ and $d$ be integers with $h \geqslant 1$ and $d \geqslant 3$, and let

$$
\begin{aligned}
& u_{i}= \begin{cases}\mathbf{0}, & i=1, \\
\boldsymbol{e}_{d}, & i=2, \\
\boldsymbol{e}_{2}+\cdots+\boldsymbol{e}_{d-1}, & i=3, \\
h\left(\boldsymbol{e}_{2}+\cdots+\boldsymbol{e}_{d-1}+\boldsymbol{e}_{d}\right), & i=4, \\
(h-1)\left(\boldsymbol{e}_{2}+\cdots+\boldsymbol{e}_{d-1}\right)+h \boldsymbol{e}_{d}, & i=5, \\
h\left(\boldsymbol{e}_{2}+\cdots+\boldsymbol{e}_{d-1}\right)+(h-1) \boldsymbol{e}_{d}, & i=6, \\
\boldsymbol{e}_{1}+4 \boldsymbol{e}_{d}, & i=7, \\
\boldsymbol{e}_{1}+5 \boldsymbol{e}_{d}, & i=8, \\
\boldsymbol{e}_{1}+\boldsymbol{e}_{2}+\cdots+\boldsymbol{e}_{d-1}, & i=9, \\
\boldsymbol{e}_{1}+\boldsymbol{e}_{2}+\cdots+\boldsymbol{e}_{d-1}+\boldsymbol{e}_{d}, & i=10,\end{cases} \\
& v_{i}=\boldsymbol{e}_{i}, \quad i=2, \ldots, d-1 \text {, } \\
& v_{i}^{\prime}=\boldsymbol{e}_{i}+\boldsymbol{e}_{d}, \quad i=2, \ldots, d-1,
\end{aligned}
$$

where $\mathbf{0}=(0, \ldots, 0) \in \mathbb{R}^{d}$ and $\boldsymbol{e}_{1}, \ldots, \boldsymbol{e}_{d}$ are the unit coordinate vectors of $\mathbb{R}^{d}$. We define the integral convex polytope $\mathcal{P}_{h, d} \subset \mathbb{R}^{d}$ by setting the convex hull of

$$
\left\{u_{1}, \ldots, u_{10}\right\} \cup\left\{v_{i}, v_{i}^{\prime}: i=2, \ldots, d-1\right\} .
$$

In this paer, we will show that $\mathcal{P}_{h, d}$ enjoys the required properties, i.e., this is a 3normal but non-normal integral convex polytope of dimension $d$ which has exactly $h$ holes. It is immediate that $\mathbb{Z}\left(\mathcal{P}_{h, d} \cap \mathbb{Z}^{d}\right)=\mathbb{Z}^{d}$. Thus $\operatorname{dim}\left(\mathcal{P}_{h, d}\right)=d$ and $\mathbb{Z} \mathcal{A}_{\mathcal{P}_{h, d}}=\mathbb{Z}^{d+1}$.

The strategy of our proof is as follows. After calculating all the facets of $\mathcal{P}_{h, d}$, we will first prove that $\mathcal{P}_{h, d} \cap\left\{\left(x_{1}, \ldots, x_{d}\right) \in \mathbb{R}^{d}: x_{1}=0\right\}$ is normal by using theory of Gröbner basis and the remaining facets of $\mathcal{P}_{h, d}$ are also normal in Section 2. In Section 3, we will analyze $2 \mathcal{P}_{h, d} \cap\left\{\left(x_{1}, \ldots, x_{d}\right) \in \mathbb{R}^{d}: x_{1}=1\right\}$ and find $h$ holes of $\mathcal{P}_{h, d}$. This implies that $\mathcal{P}_{h, d}$ is not normal. At last, Section 4 is devoted to showing that there is no hole except for such $h$ holes, also forcing $\mathcal{P}_{h, d}$ is very ample (3-normal).

\section{Normality of facets of $\mathcal{P}_{h, d}$}

In this section, we verify that the facets of $\mathcal{P}_{h, d}$ are all normal, which we shall use in Section 4.

For a hyperplane $\mathcal{H} \subset \mathbb{R}^{d}$ defined by the equality $a_{1} x_{1}+\cdots+a_{d} x_{d}=b$, we write $\mathcal{H}^{(+)}$ (resp. $\mathcal{H}^{(-)}$) for the closed half space defined by the inequality $a_{1} x_{1}+\cdots+a_{d} x_{d} \leqslant b$ (resp. $\left.a_{1} x_{1}+\cdots+a_{d} x_{d} \geqslant b\right)$. We define ten types of hyperplanes as follows: 


$$
\begin{aligned}
\mathcal{H}_{0} & : x_{1}=0, \\
\mathcal{H}_{1} & : x_{d}=0, \\
\mathcal{H}_{2, i} & :-x_{i}=0, \\
\mathcal{H}_{3, i} & :-(d-4) x_{i}+\sum_{j \neq i, 2 \leqslant j \leqslant d-1} x_{j}-x_{d}=1, \\
\mathcal{H}_{4, i}: & 4 x_{1}-4 x_{i}-x_{d}=0, \\
\mathcal{H}_{5, i}: & -4 x_{1}-x_{i}+x_{d}=1, \\
\mathcal{H}_{6, i}: & x_{1}-(d-3) x_{i}+\sum_{j \neq i, 2 \leqslant j \leqslant d-1} x_{j}=1, \\
\mathcal{H}_{7, i}: & (5 h-5) x_{1}-((d-3)(5 h-1)-4) x_{i}+(5 h-1) \sum_{j \neq i, 2 \leqslant j \leqslant d-1} x_{j}+x_{d}=5 h, \\
\mathcal{H}_{8, i}: & (h-5) x_{1}-(d-3)(h-1) x_{i}+(h-1) \sum_{j \neq i, 2 \leqslant j \leqslant d-1} x_{j}+x_{d}=h, \\
\mathcal{H}_{9, i}: & (h-1) x_{1}-((d-3) h-1) x_{i}+h \sum_{j \neq i, 2 \leqslant j \leqslant d-1} x_{j}=h,
\end{aligned}
$$

where $i=2, \ldots, d-1$. Then each hyperplane above is a supporting hyperplane of $\mathcal{P}_{h, d}$. Moreover, some routine works unable us to show that there is no facet except for the facets defined by the above $(8(d-2)+2)$ supporting hyperplanes. Hence,

$$
\mathcal{P}_{h, d}=\mathcal{H}_{0}^{(-)} \cap \mathcal{H}_{1}^{(-)} \cap\left(\bigcap_{\substack{2 \leqslant j \leqslant 9, 2 \leqslant i \leqslant d-1}} \mathcal{H}_{j, i}^{(+)}\right) .
$$

Let $\mathcal{F}_{0}, \mathcal{F}_{1}, \mathcal{F}_{j, i}$, where $j=2, \ldots, 9$ and $i=2, \ldots, d-1$, be the facets of $\mathcal{P}_{h, d}$ defined by the corresponding hyperplanes $\mathcal{H}_{0}, \mathcal{H}_{1}$ or $\mathcal{H}_{j, i}$.

We prove the normality of $\mathcal{F}_{0}$. We employ some techniques using Gröbner basis. We refer the readers to [14] for fudamental materials on Gröbner basis.

Let

$$
\begin{array}{ll}
u_{3, j}=\frac{(h-1-j) u_{3}+j u_{6}}{h-1}=(j+1)\left(\boldsymbol{e}_{2}+\cdots+\boldsymbol{e}_{d-1}\right)+j \boldsymbol{e}_{d}, & j=0,1, \ldots, h-1, \\
u_{2, j}=\frac{(h-1-j) u_{2}+j u_{5}}{h-1}=j\left(\boldsymbol{e}_{2}+\cdots+\boldsymbol{e}_{d-1}\right)+(j+1) \boldsymbol{e}_{d}, & j=0,1, \ldots, h-1, \\
u_{1, j}=\frac{(h-j) u_{1}+j u_{4}}{h}=j\left(\boldsymbol{e}_{2}+\cdots+\boldsymbol{e}_{d-1}\right)+j \boldsymbol{e}_{d}, & j=0,1, \ldots, h .
\end{array}
$$

Then $u_{3,1}, \ldots, u_{3, h-2}, u_{2,1}, \ldots, u_{2, h-2}, u_{1,1}, \ldots, u_{1, h-1}$ are all the integer points contained in $\mathcal{P}_{h, d} \cap \mathbb{Z}^{d}$ except for the vertices. Note that $u_{3,0}=u_{3}, u_{3, h-1}=u_{6}, u_{2,0}=u_{2}, u_{2, h-1}=$ $u_{5}, u_{1,0}=u_{1}$ and $u_{1, h}=u_{4}$.

Let $A_{h, d} \in \mathbb{Z}^{d \times(2(d-2)+3 h+1)}$ be an integer matrix of the form

$$
\left(v_{2}^{*}, v_{2}^{\prime *}, \cdots, v_{d-1}^{*}, v_{d-1}^{\prime *}, u_{3,0}^{*}, \ldots, u_{3, h-1}^{*}, u_{2,0}^{*}, \ldots, u_{2, h-1}^{*}, u_{1,0}^{*}, \ldots, u_{1, h}^{*}\right),
$$


where $v^{*}=\boldsymbol{e}_{1}+v$ for an integer point $v \in \mathbb{Z}^{d}$. This is nothing but a configuration arising from $\mathcal{F}_{0}$. Let $K[T]=K\left[t_{1}, t_{2}, \ldots, t_{d}\right]$ be the polynomial ring in $d$ variables over a field $K$. Then the toric ring of $A_{h, d}$ is the subalgebra $K\left[A_{h, d}\right]$ of $K[T]$ which is generated by the monomials

$$
\begin{aligned}
& t_{1} t_{2}, t_{1} t_{2} t_{d}, \ldots, t_{1} t_{d-1}, t_{1} t_{d-1} t_{d}, \quad t_{1} t_{2} \cdots t_{d-1}, t_{1} t_{2}^{2} \cdots t_{d-1}^{2} t_{d}, \ldots, t_{1} t_{2}^{h} \cdots t_{d-1}^{h} t_{d}^{h-1}, \\
& t_{1} t_{d}, t_{1} t_{2} \cdots t_{d-1} t_{d}^{2}, \ldots, t_{1} t_{2}^{h-1} \cdots t_{d-1}^{h-1} t_{d}^{h}, \quad t_{1}, t_{1} t_{2} \cdots t_{d-1} t_{d}, \ldots, t_{1} t_{2}^{h} \cdots t_{d-1}^{h} t_{d}^{h} .
\end{aligned}
$$

Let $K[X, Y, Z, W]=K\left[x_{1}, \ldots, x_{2 d-4}, y_{1}, \ldots, y_{h}, z_{1}, \ldots, z_{h}, w_{0}, w_{1}, \ldots, w_{h}\right]$ be the polynomial ring in $2 d+3 h-3$ variables over $K$ and define the surjective ring homomorphism $\pi: K[X, Y, Z, W] \rightarrow K\left[A_{h, d}\right]$ by setting

$$
\begin{array}{ll}
\pi\left(x_{2 i-1}\right)=t_{1} t_{i+1}, \pi\left(x_{2 i}\right)=t_{1} t_{i+1} t_{d} & \text { for } i=1, \ldots, d-2, \\
\pi\left(y_{j}\right)=t_{1} t_{2}^{j} \cdots t_{d-1}^{j} t_{d}^{j-1}, \pi\left(z_{j}\right)=t_{1} t_{2}^{j-1} \cdots t_{d-1}^{j-1} t_{d}^{j} & \text { for } j=1, \ldots, h, \\
\pi\left(w_{k}\right)=t_{1} t_{2}^{k} \cdots t_{d-1}^{k} t_{d}^{k} & \text { for } k=0, \ldots, h .
\end{array}
$$

The toric ideal $I$ is the kernel of the map $\pi$. Let $<$ be the lexicographic order on $K[X, Y, Z, W]$ induced by the ordering

$$
w_{h}<\cdots<w_{0}<z_{h}<\cdots<z_{1}<y_{h}<\cdots<y_{1}<x_{2 d-4}<\cdots<x_{1} .
$$

Proposition 4. A Gröbner basis of I with respect to $<$ consists of the following sets $G_{1}, \ldots, G_{8}$ of the binomials:

$$
\begin{aligned}
& G_{1}=\left\{x_{2 i-1} x_{2 j}-x_{2 i} x_{2 j-1}: 1 \leqslant i<j \leqslant d-2\right\} \\
& G_{2}=\left\{y_{i} y_{l}-y_{j} y_{k}, z_{i} z_{l}-z_{j} z_{k}: 1 \leqslant i \leqslant j \leqslant k \leqslant l \leqslant h \text { with } i+l=j+k\right\} ; \\
& G_{3}=\left\{w_{i} w_{l}-w_{j} w_{k}: 0 \leqslant i \leqslant j \leqslant k \leqslant l \leqslant h \text { with } i+l=j+k\right\} \\
& G_{4}=\left\{x_{2 i-1} z_{j}-x_{2 i} w_{j-1}, x_{2 i-1} w_{j}-x_{2 i} y_{j}: 1 \leqslant i \leqslant d-2,1 \leqslant j \leqslant h\right\} \\
& G_{5}=\left\{y_{i} z_{j}-w_{i-1} w_{j}: 1 \leqslant i, j \leqslant h\right\} ; \\
& G_{6}=\left\{y_{i} w_{j}-y_{i+1} w_{j-1}, z_{i} w_{j}-z_{i+1} w_{j-1}: 1 \leqslant i \leqslant h-1,1 \leqslant j \leqslant h\right\} ; \\
& G_{7}=\left\{x_{2 i-1} y_{j} w_{0}-x_{2 i} y_{1} y_{j-1}: 1 \leqslant i \leqslant d-2,2 \leqslant j \leqslant h\right\} ; \\
& G_{8}=\left\{\prod_{q=1}^{k} x_{2 q-1} \prod_{q=k+1}^{d-2} x_{2 q}-z_{1}^{d-4-k} z_{2} w_{0}^{k+1}: 0 \leqslant k \leqslant d-4\right\} \\
& \left.\bigcup x_{2 d-4} \prod_{q=1}^{d-3} x_{2 q-1}-w_{0}^{d-3} w_{1}, \prod_{q=1}^{d-2} x_{2 q-1}-w_{0}^{d-3} y_{1}\right\} .
\end{aligned}
$$

Proof. Let $\mathcal{G}=\bigcup_{i=1}^{8} G_{i}$. Let $\operatorname{in}_{<}\left(G_{i}\right)$ denote the set of the initial monomials of all the binomials in $G_{i}$ with respect to $<$ and $\operatorname{in}_{<}(\mathcal{G})$ the ideal generated by all the monomials in $\bigcup_{i=1}^{8} \operatorname{in}_{<}\left(G_{i}\right)$. Here the initial monomial of each binomial in $G_{i}$ is the first monomial. Since $\mathcal{G} \subset I$, we have $\operatorname{in}_{<}(\mathcal{G}) \subset \operatorname{in}_{<}(I)$. Our goal is to show $\operatorname{in}_{<}(I) \subset \operatorname{in}_{<}(\mathcal{G})$. 
Fix an irreducible non-zero binomial $f=u-v \in I$ with $v<u$. Thus $u \in \operatorname{in}_{<}(I)$. For monomials $m_{1}, m_{2} \in K[X, Y, Z, W]$, let $m_{1} \mid m_{2}$ (resp. $m_{1} \nmid m_{2}$ ) denote that $m_{2}$ is divisible (resp. not divisible) by $m_{1}$. Suppose that $u \notin \operatorname{in}_{<}(\mathcal{G})$.

First, we assume that $x_{i} \nmid u$ for any $1 \leqslant i \leqslant 2 d-4$.

- Assume that $y_{j} \nmid u$ for any $1 \leqslant j \leqslant h$. Then, for any $i, j$, both $x_{i} \nmid v$ and $y_{j} \nmid v$ are satisfied.

- When $z_{j} \nmid u$ for any $j$, the variables appearing in $u$ are only $w_{k}, 0 \leqslant k \leqslant h$, and so is $v$. Since $u-v \in I$, it must be $u \in \operatorname{in}_{<}\left(G_{3}\right)$, a contradiction.

- When $z_{j} \mid u$ for some $j$, since $u \notin \operatorname{in}_{<}\left(G_{2}\right)$, the variables among $z_{j}$ appearing in $u$ is either $z_{j}$ or $z_{j} z_{j+1}$. When the former case, i.e., when $u=z_{j}^{c_{j}} \prod w_{k}$, since $u \notin \operatorname{in}_{<}\left(G_{6}\right)$, we have $j=h$ or $\prod w_{k}=w_{0}^{d_{1}}$. If $j=h$, since $f$ is irreducible, only $w_{k}$ appears in $v$, which contradicts to $f \in I$. Similarly, if $\prod w_{k}=w_{0}^{d_{1}}$, then it contradicts $f \in I$. When the latter case, i.e., when $u=z_{j}^{c_{j}} z_{j+1}^{c_{j+1}} \prod w_{k}$, similarly, it contradicts $f \in I$.

- Assume that $y_{j} \mid u$ for some $j$. Since $u \notin \operatorname{in}_{<}\left(G_{2}\right) \cup \operatorname{in}_{<}\left(G_{5}\right) \cup \operatorname{in}_{<}\left(G_{6}\right), u$ looks like $y_{j}^{b_{j}} w_{0}^{d_{1}}, y_{j}^{b_{j}} y_{j+1}^{b_{j+1}} w_{0}^{d_{1}}$ or $y_{h}^{b_{h}} \prod w_{k}$. In these cases, similar discussions to the previous case can be applied and lead a contradiction.

Next, we assume that $x_{i} \mid u$ for some $i$.

- When only one variable $x_{i}$ appears in $u, u$ looks like $x_{i}^{a_{i}} \prod y_{j} \prod z_{j} \prod w_{k}$.

- When $i$ is even, the variables appearing in $v$ are chosen from $x_{i+1}, \ldots, x_{2 d-4}$, which obviously contradicts $f \in I$.

- When $i$ is odd, since $u \notin \operatorname{in}_{<}\left(G_{4}\right) \cup \operatorname{in}_{<}\left(G_{7}\right), u$ looks like either $x_{i}^{a_{i}} \prod y_{j}$ or $x_{i}^{a_{i}} y_{1}^{b_{1}} w_{0}^{d_{1}}$. When these cases, it contradicts $f \in I$.

- When at least $(d-1)$ distinct $x_{i}$ 's appear in $u$, there is at least one $1 \leqslant q \leqslant d-2$ such that $x_{2 q-1} x_{2 q} \mid u$, which contradicts $f \in I$.

- When there are $(d-2) x_{i}$ 's in $u$ and there is no $q$ such that $x_{2 q-1} x_{2 q} \mid u$, one has $u \in \operatorname{in}_{<}\left(G_{8}\right)$, a contradiction. When there are distinct $r x_{i}$ 's in $u$, where $2 \leqslant r \leqslant d-3$, and there is no $q$ such that $x_{2 q-1} x_{2 q} \mid u$, since $u \notin \operatorname{in}_{<}\left(G_{1}\right), u$ looks like $x_{2 i_{1}}^{a_{2 i_{1}}} \cdots x_{2 i_{l}}^{a_{2 i_{l}}} x_{2 i_{l+1}-1}^{a_{2 i_{l}+1}-1} \cdots x_{2 i_{r}-1}^{a_{2 i_{r}-1}} \prod y_{j} \prod z_{j} \prod w_{k}$, where $1 \leqslant i_{1}<\cdots<i_{r} \leqslant d-2$. This contradicts $f \in I$.

Therefore, we conclude that $u$ belongs to $\operatorname{in}_{<}(\mathcal{G})$, as required.

Corollary 5. The integral convex polytope $\mathcal{F}_{0}$ has a regular unimodular triangulation. In particular, $\mathcal{F}_{0}$ is normal.

Proof. By Proposition 4, the toric ideal $I$ has a squarefree initial ideal. This is equivalent to what $\mathcal{F}_{0}$ has a regular unimodular triangulation. (Consult, e.g., [14, Corollary 8.9].) In general, an integral convex polytope having a unimodular triangulation is normal. 
The remaining facets of $\mathcal{P}_{h, d}$ are also normal.

Lemma 6. The facets $\mathcal{F}_{1}$ and $\mathcal{F}_{j, i}$, where $j=2, \ldots, 9$ and $i=2, \ldots, d-1$, are all normal. Proof. First, let us discuss the facets $\mathcal{F}_{4, i}, \mathcal{F}_{7, i}$ and $\mathcal{F}_{8, i}$. Fix $i=2$. Then the sets of vertices of $\mathcal{F}_{4,2}, \mathcal{F}_{7,2}$ and $\mathcal{F}_{8,2}$ are $\left\{u_{1}, u_{7}, u_{9}, v_{3}, \ldots, v_{d-1}\right\},\left\{u_{4}, u_{8}, u_{10}, v_{3}^{\prime}, \ldots, v_{d-1}^{\prime}\right\}$ and $\left\{u_{4}, u_{5}, u_{8}, v_{3}^{\prime}, \ldots, v_{d-1}^{\prime}\right\}$, respectively. Each of the matrices whose column vectors are the vertices of each facet can be transformed into the matrix $\left(\mathbf{0}, \boldsymbol{e}_{1}, \ldots, \boldsymbol{e}_{d-1}\right)$ by unimodular transformations. Thus each facet $\mathcal{F}_{4,2}, \mathcal{F}_{7,2}$ or $\mathcal{F}_{8,2}$ is unimodularly equivalent to a unit simplex of dimension $d-1$. Thus, in particular, $\mathcal{F}_{4,2}, \mathcal{F}_{7,2}$ and $\mathcal{F}_{8,2}$ are normal. Similarly, for any $i, \mathcal{F}_{4, i}, \mathcal{F}_{7, i}$ and $\mathcal{F}_{8, i}$ are normal.

Next, let us investigate $\mathcal{F}_{1}, \mathcal{F}_{2, i}, \mathcal{F}_{6, i}$ and $\mathcal{F}_{9, i}$. For $\mathcal{F}_{2,2}$, the set of its vertices is

$$
\left\{u_{1}, u_{2}, u_{7}, u_{8}, v_{3}, \ldots, v_{d-1}, v_{3}^{\prime}, \ldots, v_{d-1}^{\prime}\right\} .
$$

By unimodular transformations, the matrix whose column vector is the above vertices can be transformed into the matrix

$$
\left(0,-e_{d}, e_{1}, e_{1}-e_{d}, e_{3}, \ldots, e_{d-1}, e_{3}-e_{d}, \ldots, e_{d-1}-e_{d}\right) .
$$

This is totally unimodular ([13, Chapter 19]). Thus, $\mathcal{F}_{2,2}$ has a unimodular triangulation. In particular, this is normal. Similarly, for any $i, \mathcal{F}_{2, i}$ is normal and so are $\mathcal{F}_{1}, \mathcal{F}_{6, i}$ and $\mathcal{F}_{9, i}$.

Finally, let us consider the facets $\mathcal{F}_{3, i}$ and $\mathcal{F}_{5, i}$. Then one can see that each of them is unimodularly equivalent to the simplex whose vertex set is $\left\{\mathbf{0}, \boldsymbol{e}_{1}, \ldots, \boldsymbol{e}_{d-2},(h-1) \boldsymbol{e}_{d-1}\right\}$. This is also normal, as desired.

\section{Holes of $\mathcal{P}_{h, d}$}

In this section, we find $h$ holes of $\mathcal{P}_{h, d}$. Let

$$
u_{j}^{\prime}=\frac{1}{2}\left(u_{1, j-1}+u_{1, j}+u_{8}+u_{9}\right) \text { for } j=1, \ldots, h .
$$

Then $u_{j}^{\prime}=\boldsymbol{e}_{1}+j\left(\boldsymbol{e}_{2}+\cdots+\boldsymbol{e}_{d-1}\right)+(j+2) \boldsymbol{e}_{d}$ and each $\left(u_{j}^{\prime}, 2\right)$ is contained in $\mathbb{R}_{\geqslant 0} \mathcal{A}_{\mathcal{P}_{h, d}} \cap \mathbb{Z}^{d+1}$. On the other hand, since none of the points $\left(u_{j}^{\prime}, 2\right)-\left(u_{i}, 1\right)$, where $i=7, \ldots, 10$, are contained in $\mathcal{A}_{\mathcal{P}_{h, d}}$, it must be $\left(u_{j}^{\prime}, 2\right) \notin \mathbb{Z}_{\geqslant 0} \mathcal{A}_{\mathcal{P}_{h, d}}$. Hence, the above $h$ integer points are holes of $\mathcal{P}_{h, d}$. In the rest of this section, we show that there is no more holes in

$$
\left\{x \in \mathbb{R}_{\geqslant 0} \mathcal{A}_{\mathcal{P}_{h, d}} \cap \mathbb{Z}^{d+1}: \operatorname{deg}(x)=2\right\} .
$$

For nonnegative integers $n$ and $k$, let

$$
\mathcal{P}_{h, d}(n, k)=n \mathcal{P}_{h, d} \cap\left\{\left(x_{1}, \ldots, x_{d}\right) \in \mathbb{R}^{d}: x_{1}=k\right\} .
$$

For example, $\mathcal{P}_{h, d}(1,0)=\mathcal{F}_{0}$ and $\mathcal{P}_{h, d}(1,1)=\operatorname{conv}\left(\left\{u_{7}, u_{8}, u_{9}, u_{10}\right\}\right)$. Let $\mathcal{P}_{0}=\mathcal{P}_{h, d}(1,0)$ and $\mathcal{P}_{1}=\mathcal{P}_{h, d}(1,1)$.

For a hyperplane $\mathcal{H}$ defined by the equality $a_{1} x_{1}+\cdots+a_{d} x_{d}=b$ and a positive integer $m$, we write $m \mathcal{H}^{(+)}$(resp. $m \mathcal{H}^{(-)}$) for the closed half space defined by the inequality $a_{1} x_{1}+\cdots+a_{d} x_{d} \leqslant m b\left(\right.$ resp. $\left.a_{1} x_{1}+\cdots+a_{d} x_{d} \geqslant m b\right)$. 
Lemma 7. Let $\mathcal{Q}=\mathcal{P}_{h, d}(2,1)$. Then one has

$$
\mathcal{Q} \cap \mathbb{Z}^{d}=\left\{\mathbf{a}_{\mathbf{0}}+\mathbf{a}_{\mathbf{1}} \in \mathbb{Z}^{d}: \mathbf{a}_{\mathbf{i}} \in \mathcal{P}_{i} \cap \mathbb{Z}^{d}, i=0,1\right\} \cup\left\{u_{1}^{\prime}, \ldots, u_{h}^{\prime}\right\} .
$$

Proof. Clearly, $\mathcal{Q} \cap \mathbb{Z}^{d} \supset\left\{\mathbf{a}_{\mathbf{0}}+\mathbf{a}_{\mathbf{1}} \in \mathbb{Z}^{d}: \mathbf{a}_{\mathbf{i}} \in \mathcal{P}_{i} \cap \mathbb{Z}^{d}, i=0,1\right\} \cup\left\{u_{1}^{\prime}, \ldots, u_{h}^{\prime}\right\}$. Thus it is enough to show the other inclusion. We remark that from (1), one has

$$
\mathcal{Q} \subset 2 \mathcal{H}_{0}^{(-)} \cap 2 \mathcal{H}_{1}^{(-)} \cap\left(\bigcap_{\substack{2 \leqslant j \leqslant 9, 2 \leqslant i \leqslant d-1}} 2 \mathcal{H}_{j, i}^{(+)}\right) .
$$

Let $x=\left(1, x_{2}, \ldots, x_{d}\right) \in \mathcal{Q} \cap \mathbb{Z}^{d}$.

The first step. Assume that $x_{2}=x_{3}=\cdots=x_{d-1}$. Since $x \in 2 \mathcal{H}_{2,2}^{(+)} \cap 2 \mathcal{H}_{9,2}^{(+)}$, one has $0 \leqslant x_{2} \leqslant h+1$. On the other hand, since

$$
x \in \bigcap_{\substack{j=3,4,5,7,8, 2 \leqslant i \leqslant d-1}} 2 \mathcal{H}_{j, i}^{(+)},
$$

we have

$$
\max \left\{x_{2}-2,-4\left(x_{2}-1\right)\right\} \leqslant x_{d} \leqslant \min \left\{x_{2}+6,5 h+5-4 x_{2}, h+5\right\} .
$$

One can verify that all of these are contained in $\left\{\mathbf{a}_{\mathbf{0}}+\mathbf{a}_{\mathbf{1}} \in \mathbb{Z}^{d}: \mathbf{a}_{\mathbf{i}} \in \mathcal{P}_{i} \cap \mathbb{Z}^{d}, i=\right.$ $0,1\} \cup\left\{u_{1}^{\prime}, \ldots, u_{h}^{\prime}\right\}$.

The second step. Assume that $x$ does not satisfy $x_{2}=x_{3}=\cdots=x_{d-1}$. Let $a_{1}, \ldots, a_{m}$ be distinct $m$ integers such that $\left\{a_{1}, \ldots, a_{m}\right\}=\left\{x_{2}, \ldots, x_{d-1}\right\}$, where $a_{1}>a_{2}>\cdots>a_{m}$. Then $m \geqslant 2$. By $x \in \bigcap_{i=2}^{d-1} 2 \mathcal{H}_{2, i}^{(+)}$, we have $a_{m} \geqslant 0$. Let $p_{\ell}$ be the number of $a_{\ell}$ 's among $x_{2}, \ldots, x_{d-1}$. Thus, $p_{\ell}>0$ and $p_{1}+\cdots+p_{m}=d-2$. For $\ell=1, \ldots, m-1$, let $b_{\ell}=a_{\ell}-a_{m}$. Then $b_{\ell} \geqslant m-\ell$. From $x \in \bigcap_{i=2}^{d-1} 2 \mathcal{H}_{6, i}^{(+)}$, we have

$$
\begin{aligned}
& -(d-3) a_{m}+\left(p_{1} a_{1}+\cdots+p_{m-1} a_{m-1}+\left(p_{m}-1\right) a_{m}\right) \\
& \quad=-(d-3) a_{m}+\left(p_{1}\left(a_{m}+b_{1}\right)+\cdots+p_{m-1}\left(a_{m}+b_{m-1}\right)+\left(p_{m}-1\right) a_{m}\right) \\
& \quad=-(d-2) a_{m}+\left(p_{1}+\cdots+p_{m}\right) a_{m}+p_{1} b_{1}+\cdots+p_{m-1} b_{m-1} \\
& \quad=p_{1} b_{1}+\cdots+p_{m-1} b_{m-1} \leqslant 1 .
\end{aligned}
$$

Hence, we obtain $m=2$ and $p_{1}=b_{1}=1$. Let, say, $x=\left(1, a_{m}+1, a_{m}, \ldots, a_{m}, x_{d}\right)$. Moreover, from $x \in \bigcap_{i=2}^{d-1} 2 \mathcal{H}_{9, i}^{(+)}$, we have

$$
-((d-3) h-1) a_{m}+h\left(a_{m}+1+(d-4) a_{m}\right)=h+a_{m} \leqslant h+1,
$$

which implies that $a_{m}=0$ or 1 .

- When $x=\left(1,1,0, \ldots, 0, x_{d}\right)$, since $x \in 2 \mathcal{H}_{4,3}^{(+)} \cap 2 \mathcal{H}_{5,3}^{(+)}$, we have $4 \leqslant x_{d} \leqslant 6$.

- When $x=\left(1,2,1, \ldots, 1, x_{d}\right)$, since $x \in 2 \mathcal{H}_{1}^{(-)} \cap 2 \mathcal{H}_{7,3}^{(+)}$, we have $0 \leqslant x_{d} \leqslant 2$. 
All of these are contained in $\left\{\mathbf{a}_{\mathbf{0}}+\mathbf{a}_{\mathbf{1}} \in \mathbb{Z}^{d}: \mathbf{a}_{\mathbf{i}} \in \mathcal{P}_{i} \cap \mathbb{Z}^{d}, i=0,1\right\}$. Similarly, the integer points $x=\left(1, a_{m}, \ldots, a_{m}, x_{d}\right)+\boldsymbol{e}_{j}$, where $a_{m} \in\{0,1\}$ and $j=3, \ldots, d-1$, are also contained there, as required.

Now, Corollary 5 says that $\mathcal{P}_{0}$ is normal. Moreover, since $\mathcal{P}_{1}$ is of dimension 2 , this is also normal ([6, Corollary 2.2.13]). Thus, there is no hole in

$$
\left\{\left(x_{1}, \ldots, x_{d}, x_{d+1}\right) \in \mathbb{R}_{\geqslant 0} \mathcal{A}_{\mathcal{P}_{h, d}} \cap \mathbb{Z}^{d+1}: x_{1} \in\{0,2\}, x_{d+1}=2\right\} .
$$

Therefore, there exist exactly $h$ holes contained in (2).

\section{The 3-normality of $\mathcal{P}_{h, d}$}

In this section, we claim that there is no other hole except for $\left(u_{j}^{\prime}, 2\right), j=1, \ldots, h$. In other words, we prove that $\mathcal{P}_{h, d}$ is 3-normal.

Similar computations to Lemma 7 enable us to show the following

Lemma 8. One has

(a) $\mathcal{P}_{h, d}(3,1) \cap \mathbb{Z}^{d}=\left\{\mathbf{a}_{\mathbf{0}}+\mathbf{a}_{\mathbf{0}}{ }^{\prime}+\mathbf{a}_{\mathbf{1}} \in \mathbb{Z}^{d}: \mathbf{a}_{\mathbf{0}}, \mathbf{a}_{\mathbf{0}}{ }^{\prime} \in \mathcal{P}_{0} \cap \mathbb{Z}^{d}, \mathbf{a}_{\mathbf{1}} \in \mathcal{P}_{1} \cap \mathbb{Z}^{d}\right\} ;$

(b) $\mathcal{P}_{h, d}(3,2) \cap \mathbb{Z}^{d}=\left\{\mathbf{a}_{\mathbf{0}}+\mathbf{a}_{\mathbf{1}}+\mathbf{a}_{\mathbf{1}}{ }^{\prime} \in \mathbb{Z}^{d}: \mathbf{a}_{\mathbf{0}} \in \mathcal{P}_{0} \cap \mathbb{Z}^{d}, \mathbf{a}_{\mathbf{1}}, \mathbf{a}_{\mathbf{1}}{ }^{\prime} \in \mathcal{P}_{1} \cap \mathbb{Z}^{d}\right\}$;

(c) $\mathcal{P}_{h, d}(4,1) \cap \mathbb{Z}^{d}=\left\{\mathbf{a}_{\mathbf{0}}+\mathbf{a}_{\mathbf{0}}{ }^{\prime}+\mathbf{a}_{\mathbf{0}}{ }^{\prime \prime}+\mathbf{a}_{\mathbf{1}} \in \mathbb{Z}^{d}: \mathbf{a}_{\mathbf{0}}, \mathbf{a}_{\mathbf{0}}{ }^{\prime}, \mathbf{a}_{\mathbf{0}}{ }^{\prime \prime} \in \mathcal{P}_{0} \cap \mathbb{Z}^{d}, \mathbf{a}_{\mathbf{1}} \in \mathcal{P}_{1} \cap \mathbb{Z}^{d}\right\}$.

Finally, we prove

Lemma 9. Let $n \geqslant 3$ and $0 \leqslant k \leqslant n$. For each $\alpha \in \mathcal{P}_{h, d}(n, k)$, we have

$$
\alpha=\mathbf{a}_{\mathbf{0}}{ }^{(1)}+\cdots+\mathbf{a}_{\mathbf{0}}{ }^{(n-k)}+\mathbf{a}_{\mathbf{1}}{ }^{(1)}+\cdots+\mathbf{a}_{\mathbf{1}}{ }^{(k)},
$$

where $\mathbf{a}_{\mathbf{0}}{ }^{(s)} \in \mathcal{P}_{0} \cap \mathbb{Z}^{d}$ for $s=1, \ldots, n-k$ and $\mathbf{a}_{\mathbf{1}}{ }^{(t)} \in \mathcal{P}_{1} \cap \mathbb{Z}^{d}$ for $t=1, \ldots, k$. That is to say, $\mathcal{P}_{h, d}$ is 3-normal.

Proof. Fix $\alpha=\left(\alpha_{1}, \ldots, \alpha_{d}\right) \in \mathcal{P}_{h, d}(n, k)$, where $\alpha_{1}=k$. Since $\mathcal{P}_{0}$ and $\mathcal{P}_{1}$ are normal, we may assume that $1 \leqslant k \leqslant n-1$. Moreover, thanks to Lemma 8 , we may also assume that $n \geqslant 4, k \geqslant 2$ or $n \geqslant 5, k=1$. In addition, by Lemma 6 , we may also assume that

$$
\alpha \notin n \mathcal{F}_{0} \cup n \mathcal{F}_{1} \cup\left(\bigcup_{\substack{2 \leqslant j \leqslant 9, 2 \leqslant i \leqslant d-1}} n \mathcal{F}_{j, i}\right) \text {. }
$$

We will proceed our discussions by induction on $n$.

The first step. Suppose that $\alpha$ satisfies the following $(d-1)$ inequalities:

$$
\alpha_{d} \geqslant 5 \text { and }-(d-4) \alpha_{i}+\sum_{j \neq i, 2 \leqslant j \leqslant d-1} \alpha_{j}-\alpha_{d} \leqslant n-6 \text { for } i=2, \ldots, d-1 .
$$

Let $\beta=\alpha-u_{8}=\left(\alpha_{1}-1, \alpha_{2}, \ldots, \alpha_{d-1}, \alpha_{d}-5\right)$. Then we have $\beta \in \mathcal{P}_{h, d}(n-1, k-1) \cap \mathbb{Z}^{d}$. In fact, one can easily see that for $i=2, \ldots, d-1$, we have 
- $\alpha_{1}-1=k-1 \geqslant 0$;

- $\alpha_{d}-5 \geqslant 0$ by (4);

- $\alpha_{i} \geqslant 0$;

- $-(d-4) \alpha_{i}+\sum_{j \neq i, 2 \leqslant j \leqslant d-1} \alpha_{j}-\left(\alpha_{d}-5\right) \leqslant n-6+5=n-1$ by $(4)$;

- $4\left(\alpha_{1}-1\right)-4 \alpha_{i}-(\alpha-5) \leqslant-1-4+5=0$ since $\alpha \notin n \mathcal{F}_{4, i}$;

- $-4\left(\alpha_{1}-1\right)-\alpha_{i}+\alpha_{d}-5 \leqslant n-1$;

- $\alpha_{1}-1-(d-3) \alpha_{i}+\sum_{j \neq i, 2 \leqslant j \leqslant d-1} \alpha_{j} \leqslant n-1$;

- $(5 h-5)\left(\alpha_{1}-1\right)-((d-3)(5 h-1)-4) \alpha_{i}+(5 h-1) \sum_{j \neq i, 2 \leqslant j \leqslant d-1} \alpha_{j}+\alpha_{d}-5 \leqslant$ $5 h n-(5 h-5)-5=5 h(n-1)$;

- $(h-5)\left(\alpha_{1}-1\right)-(d-3)(h-1) \alpha_{i}+(h-1) \sum_{j \neq i, 2 \leqslant j \leqslant d-1} \alpha_{j}+\alpha_{d}-5 \leqslant h n-(h-5)-5=$ $h(n-1)$;

- $(h-1)\left(\alpha_{1}-1\right)-((d-3) h-1) \alpha_{i}+h \sum_{j \neq i, 2 \leqslant j \leqslant d-1} \alpha_{j} \leqslant h n-1-(h-1)=h(n-1)$ since $\alpha \notin n \mathcal{F}_{9, i}$.

The above estimations imply that $\beta \in(n-1) \mathcal{P}_{h, d} \cap \mathbb{Z}^{d}$ because of (1). By the hypothesis of induction, we obtain the required expression on $\alpha$ like (3).

The second step. Suppose that $\alpha$ satisfies either

$$
\alpha_{d} \leqslant 4 \text { or }-(d-4) \alpha_{i}+\sum_{\substack{j \neq i, 2 \leqslant j \leqslant d-1}} \alpha_{j}-\alpha_{d} \geqslant n-5 \text { for } i=2, \ldots, d-1 .
$$

Then we obtain the new inequalities

$$
\begin{aligned}
& -4 \alpha_{1}-\alpha_{i}+\alpha_{d} \leqslant n-6, \\
& (h-5) \alpha_{1}-(d-3)(h-1) \alpha_{i}+(h-1) \sum_{j \neq i, 2 \leqslant j \leqslant d-1} \alpha_{j}+\alpha_{d} \leqslant h n-5
\end{aligned}
$$

for $i=2, \ldots, d-1$ as follows.

(i) First, suppose that $\alpha$ satisfies the left-hand condition of (5). Since $\alpha \notin n \mathcal{F}_{2, i}$, one has $\alpha_{i} \geqslant 1 \geqslant 10-4 k-n$ from our assumption $n \geqslant 4, k \geqslant 2$ or $n \geqslant 5, k=1$. Thus we obtain

$$
-4 \alpha_{1}-\alpha_{i}+\alpha_{d} \leqslant-4 k-10+4 k+n+4=n-6 .
$$

Moreover, since $\alpha \notin n \mathcal{F}_{6, i}$, one has $\alpha_{1}-(d-3) \alpha_{i}+\sum_{j \neq i, 2 \leqslant j \leqslant d-1} \alpha_{j} \leqslant n-k-1$. Hence $(h-1)\left(\alpha_{1}-(d-3) \alpha_{i}+\sum_{j \neq i, 2 \leqslant j \leqslant d-1} \alpha_{j}\right) \leqslant(h-1)(n-1)+4 k+h+n-10$. Remark that $h \geqslant 1$. Thus we also obtain

$$
\begin{aligned}
& (h-5) \alpha_{1}-(d-3)(h-1) \alpha_{i}+(h-1) \sum \alpha_{j}+\alpha_{d} \\
& \leqslant-4 \alpha_{1}+(h-1)(n-1)+4 k+h+n-10+4=h n-5 .
\end{aligned}
$$


(ii) Second, suppose that $\alpha$ satisfies the right-hand condition of (5). Since $\alpha \notin n \mathcal{F}_{6, i}$, one has $-4 \alpha_{1}-(d-3) \alpha_{i}+\sum \alpha_{j} \leqslant n-5 k-1 \leqslant n-5 k-1+n+5 k-10=2 n-11$. Thus we obtain

$$
\begin{aligned}
-4 \alpha_{1}-\alpha_{i}+\alpha_{d} & =-4 \alpha_{1}-(d-3) \alpha_{i}+\sum \alpha_{j}-\left(-(d-4) \alpha_{i}+\sum \alpha_{j}-\alpha_{d}\right) \\
& \leqslant 2 n-11-n+5=n-6 .
\end{aligned}
$$

Moreover, since $\alpha \notin n \mathcal{F}_{9, i}$, one has $(h-1) \alpha_{1}-((d-3) h-1) \alpha_{i}+h \sum \alpha_{j} \leqslant h n-1 \leqslant$ $h n-1+4 k+n-9=h n+4 k+n-10$. Thus we obtain

$$
\begin{aligned}
& (h-5) \alpha_{1}-(d-3)(h-1) \alpha_{i}+(h-1) \sum \alpha_{j}+\alpha_{d} \\
& \quad=-4 \alpha_{1}+(h-1) \alpha_{1}-((d-3) h-1) \alpha_{i}+h \sum \alpha_{j}-\left(-(d-4) \alpha_{i}+\sum \alpha_{j}-\alpha_{d}\right) \\
& \quad \leqslant-4 k+h n+4 k+n-10-n+5=h n-5 .
\end{aligned}
$$

Let $\beta^{\prime}=\alpha-u_{9}$. If we assume that $\alpha$ satisfies (5) then similar to the first step, we can verify that $\beta^{\prime} \in(n-1) \mathcal{P}_{h, d} \cap \mathbb{Z}^{d}$. Here we use (6) and the normality of some facets of $\mathcal{P}_{h, d}$ in the same way as the first step.

The third step. Suppose that $\alpha$ satisfies neither (4) nor (5). When this is the case, one has $d \geqslant 4$ and there exist $\ell$ and $\ell^{\prime}$ with $2 \leqslant \ell \neq \ell^{\prime} \leqslant d-1$ such that the inequalities

$$
-(d-4) \alpha_{\ell}+\sum \alpha_{j}-\alpha_{d} \leqslant n-6 \text { and }-(d-4) \alpha_{\ell^{\prime}}+\sum \alpha_{j}-\alpha_{d} \geqslant n-5
$$

are satisfied. It then follows that $(d-3)\left(\alpha_{\ell}-\alpha_{\ell^{\prime}}\right) \geqslant 1$, i.e., $\alpha_{\ell}-\alpha_{\ell^{\prime}} \geqslant 1$. Let $\beta^{\prime \prime}=\alpha-v_{\ell}$. Then, similarly, we can verify that $\beta^{\prime \prime} \in(n-1) \mathcal{P}_{h, d} \cap \mathbb{Z}^{d}$ by using $\alpha_{\ell}-\alpha_{\ell^{\prime}} \geqslant 1$ and the normality of some facets of $\mathcal{P}_{h, d}$, as desired.

\section{References}

[1] M. Beck, J. Delgado and J. Gubeladze, Very ample and Koszul segmental fibrations, arXiv:1307.7422v2.

[2] T. Bogart, C. Haase, M. Hering, B. Lorenz, B. Nill, A. Paffenholz, F. Santos and H. Schenck, Few smooth $d$-polytopes with $N$ lattice points, arXiv:1010.3887v1.

[3] W. Bruns, The quest for counterexamples in toric geometry, arXiv:1110.1840v1.

[4] W. Bruns and J. Gubeladze, Polytopal linear groups, J. Algebra, 219 (1999), 715737.

[5] W. Bruns and J. Gubeladze, "Polytopes, rings and K-theory," Springer-Verlag, Heidelberg, 2009.

[6] D. Cox, J. Little and H. Schenck, "Toric varieties," American Mathematical Society, 2011.

[7] T. Hibi, A. Higashitani, L. Katthän and R. Okazaki, Normal cyclic polytopes and non-very ample cyclic polytopes, J. Australian Math. Soc., to appear. 
[8] S. Ogata, On projective toric varieties whose defining ideals have minimal generators of the highest degree, Annales de L'Institut Fourier 53 (2003), 2243-2255.

[9] S. Ogata, Very ample but not normal lattice polytopes, Beitr. Algebra Geom. 54 (2013), 291-302.

[10] S. Ogata and K. Nakagawa, On generators of ideals defining projective toric varieties, Manuscripta Math. 108 (2002), 33-42.

[11] H. Ohsugi and T. Hibi, Normal polytopes arising from finte graphs, J. Algebra 207 (1998), 409-426.

[12] H. Ohsugi and T. Hibi, Non-very ample configurations arising from contingency tables, Annals of the Institute of Statistical Math. 62 (2010), 639-644.

[13] A. Schrijver, "Theory of Linear and Integer Programming," John Wiley \& Sons, 1986.

[14] B. Sturmfels, "Gröbner Bases and Convex Polytopes," volume 8 of University Lecture Series, American Mathematical Society, Providence, RI, 1995. 